\title{
Evaluation Index for Preventive Maintenance of Highway Asphalt Pavement
}

\author{
Weiling Teng ${ }^{1 *}$, Xiaofang $\mathrm{Li}^{1}$ \\ ${ }^{1}$ College of Civil Engineering, Xijing University, Xi 'an, Shaanxi
}

\begin{abstract}
In order to make the decision of preventive maintenance of asphalt pavement, this paper analyses the related research, and finds the problems in the evaluation index system of preventive maintenance. Based on the characteristics of asphalt pavement and the actual maintenance demand, the evaluation index system of preventive maintenance of asphalt pavement is constructed.
\end{abstract}

\section{Introduction}

Pavement prevention maintenance is an active protection project taken in advance. Preventive maintenance can effectively prevent the diseases, slow down the deterioration, and extend service life. Preventive maintenance can alleviate the financial pressure of maintenance departments, which has important economic and social significance ${ }^{[1]}$. Preventive maintenance should follow the principle of scientific decision-making and prevention first. The focus of preventive maintenance is the pavement with good overall performance but slight diseases. Therefore, it is particularly important to make scientific and reasonable timing selection and evaluation criteria for preventive maintenance.

The Technical Specification for Maintenance of Asphalt Pavement (JTG 5142-2019) stipulates that the section of asphalt pavement technical Condition index (PQI) and its sub-indexes are evaluated as "excellent and good", and daily maintenance, prevention maintenance or repair maintenance can be carried out. ${ }^{[2]}$, the evaluation standard could not distinguish between daily maintenance and preventive maintenance and lacked practical guiding significance; According to the Highway Asphalt Pavement Maintenance Design Code (JTG 5421-2018), each evaluation unit can be divided into maintenance types such as prevention maintenance and repair maintenance by using pavement technical condition data. However, the classification method is too complicated and the operation is relatively complex. Many researchers are adapted to the preventive maintenance of the evaluation index system and standard for more detailed analysis, but it has not yet formed a mature domestic pavement preventive maintenance evaluation standard, generally determined according to experience in maintenance, preventive maintenance and key points in the "prevention", should be based on technology of grade, use fixed number of year, road conditions, traffic volume size and composition, factors such as climate condition, to determine asphalt pavement preventive maintenance time ${ }^{[2]}$.
Only the single or comprehensive performance index of asphalt pavement is used as the evaluation index, but the concept of "average" exists in the comprehensive index of road performance evaluation. When a certain disease is more serious and other aspects are in good condition, the overall evaluation is better after average treatment, but actually preventive maintenance is needed. Therefore, further research is needed on the evaluation index suitable for preventive maintenance. For scientific decision-making and the priority of the asphalt pavement preventive maintenance, the best time to reasonable judgment of asphalt pavement preventive maintenance and preventive maintenance quantitative guidance specific measures, this paper studies the evaluation index of asphalt pavement preventive maintenance system, based on the analysis of the current evaluation index of asphalt pavement, according to the characteristics of the asphalt pavement preventive maintenance, build the evaluation index system.

\section{Evaluation for preventive maintenance in specifications}

In the relevant specifications, pavement condition index (PQI) and each sub-index are generally taken as the evaluation criteria of maintenance scheme, which are summarized as follows in three aspects.

\subsection{Evaluation indexes and standards for pavement technical conditions}

Maintenance project plan and maintenance countermeasures should be formulated according to the investigation and evaluation results of each sub-index of asphalt pavement technical condition index (PQI).In the Technical Specification for Highway Asphalt Pavement Maintenance, sections with the technical Condition index (PQI) of asphalt pavement and each sub-index are evaluated as "excellent and good" can be maintained daily, 
prevented or repaired. Sections with any sub-index evaluated as "medium" or below should be repaired and maintained. According to the Highway Technical Condition Evaluation Standard, taking the expressway as an example, the grade standard of "excellent and good" pavement is shown in Table 1.

Table 1. Classification of highway Technical Conditions.

\begin{tabular}{|c|c|c|}
\hline Evaluation index & Optimal & Good \\
\hline PQI & $\geq 90$ & $\geq 80,<90$ \\
\hline RQI, RDI, PBI, PWI, SRI, PSSI & $\geq 90$ & $\geq 80,<90$ \\
\hline PCI & $\geq 92$ & $\geq 80,<92$ \\
\hline
\end{tabular}

According to the Technical Specification for Maintenance of Asphalt Pavement, the technical condition index of asphalt pavement should meet the requirements, as shown in Table 2. If the requirements are not met, a reasonable maintenance plan should be arranged and comprehensive maintenance measures should be taken.

Table 2. Technical requirements for highway asphalt pavement.

\begin{tabular}{|c|c|c|c|c|c|c|}
\hline \multirow{2}{*}{$\begin{array}{c}\text { Technical Performance } \\
\text { Index }\end{array}$} & $\begin{array}{c}\text { Network } \\
\text { level }\end{array}$ & \multicolumn{5}{|c|}{ Basic unit } \\
\cline { 2 - 7 } & PQI & PQI & PCI & RQI & RDI & SRI \\
\hline Highway & $\geq 90$ & $\geq 80$ & $\geq 80$ & $\geq 80$ & $\geq 75$ & $\geq 75$ \\
\hline
\end{tabular}

\subsection{Standard of preventive maintenance measures for asphalt pavement}

According to the Technical Specification for Maintenance of Highway Asphalt Pavement, the application of premaintenance measures such as sealing layer, micrometer and cover is shown in Table 3.

\subsection{Pavement technical condition data index}

According to the Highway Asphalt Pavement Maintenance Design Code, after the evaluation and analysis of the evaluation units based on the pavement technical status data, the evaluation units can be divided into preventive maintenance level repair maintenance and other maintenance types, as shown in Table 4.

Table 3. Pre-maintenance Technology and Road Performance Level (Highway).

\begin{tabular}{|c|c|c|}
\hline Mesures & $\begin{array}{c}\text { Road conditions } \\
\text { index }\end{array}$ & Highway \\
\hline \multirow{2}{*}{ Fog Sealing Layer } & PCI, RQI, RDI & $\geq 90$ \\
\cline { 2 - 3 } & SRI & $\geq 75$ \\
\hline
\end{tabular}

\begin{tabular}{|c|c|c|}
\hline Micro-Surfacing & PCI, RQI & $\geq 85$ \\
\hline $\begin{array}{c}\text { Composite sealing } \\
\text { layer }\end{array}$ & PCI, RQI, RDI & $\geq 80$ \\
\hline \multirow{2}{*}{ Ultra-thin surface } & PCI, RQI & $\geq 85$ \\
\cline { 2 - 3 } & RDI & $\geq 80$ \\
\hline \multirow{2}{*}{ Thin overlay } & PCI, RQI & $\geq 80$ \\
\cline { 2 - 3 } & RDI & $\geq 75$ \\
\hline Overlay & PCI, RQI & $\geq 80$ \\
\hline
\end{tabular}

Table 4. Evaluation method of unit maintenance type division.

\begin{tabular}{|c|c|c|c|c|}
\hline \multicolumn{4}{|c|}{ Evaluation Index } & \multirow{2}{*}{ Maintenance type } \\
\hline PCI & RQI & RDI & SRI & \\
\hline \multirow{4}{*}{$\geq 90$} & \multirow{2}{*}{$\geq 90$} & $\geq 80$ & $<75$ & Preventive \\
\hline & & $<80$ & & Repair \\
\hline & $85-90$ & & & Preventive \\
\hline & $<85$ & & & Repair \\
\hline \multirow{2}{*}{$85-90$} & $\geq 85$ & & & Preventive \\
\hline & $<85$ & & & Repair \\
\hline$<85$ & & & & Repair \\
\hline
\end{tabular}

In conclusion, in the relevant rules and regulations, generally based on pavement condition index (PQI) and each component index as an evaluation standard maintenance plan, but the PQI scope is not unified, comprehensive, PQI and each component index is larger than 80 or 85 above, can carry out preventive maintenance, obviously, the current evaluation standards is too general, and did not form a unified index system and practical operation is difficult.

\section{Pre-maintenance evaluation index of asphalt pavement in relevant studies}

Many domestic researchers have analyzed and studied the evaluation index system and standard of the preventive maintenance of asphalt pavement on the basis of the current specifications.

Guangzhou ShiYan expressway co., LTD, with good strength of pavement structure and above (SSI acuity 0.85 ) as the basic premise, on the basis of comprehensive index of pavement performance, increase the crack rate of CR, rut $\mathrm{RD}$, anti-sliding performance of SFC, flatness IRI four single index, specific criteria for $\mathrm{PQI}>92, \mathrm{CR}<0.5 \%$, $\mathrm{RD}<5 \mathrm{~mm}$, SFC $>50$, IRI 2 or less 0 , preventive maintenance should be considered[3]; Based on the existing maintenance specifications, the evaluation index 
was expanded and refined, and the evaluation standard improved the sensitivity of disease recognition.

Xuying Fan Hebei construction engineering college, etc., in the traditional performance and asphalt pavement material performance as a two level of evaluation indexes, and puts forward the three secondary evaluation index of asphalt material performance under, the thermal properties, tar, asphalt performance at low temperature, high temperature performance and using the improved entropy weight of the analytic hierarchy process to determine each index weight ${ }^{[4]}$; However, the evaluation criteria were not studied. For different regions or new road sections, they had to be re-assigned according to their environmental requirements for calculation.

China highway engineering consulting group co., LTD., marshal dong, etc, put forward the road conditions trigger method is used to determine preventive maintenance time, on the basis of traditional effect - cost than, put forward the effect ratio and energy consumption effects - carbon emissions than two new evaluation indexes, using life cycle 3 indexes of maximum value to determine preventive maintenance time, it is concluded that the best opportunity to implement the micro table place maintenance for $\mathrm{PCI}=90^{[5]}$.

Chunan Zhang second highway engineering co., LTD., etc., put forward from the aspects of technology, environment and economy three indicators establish preventive maintenance of macroscopic and microscopic evaluation system, the cost - benefit cost evaluation criterion, building the appraisal model of asphalt pavement preventive maintenance decision-making, according to the plan layer selected 10 kinds of typical curing technology index matrix model is established and the forecast of cost - benefit of index weight calculation, the preventive maintenance program ${ }^{[6]}$.

\section{Construction of preventive maintenance evaluation index system}

In current specification and related research, damaged pavement condition index PCI was adopted as a judge maintenance evaluation index, but PCI is comprehensive, includes the deformation, cracks, loose kind of diseases, it cannot accurately reflect the road, under the condition of single disease and preventive maintenance technology focuses on a single disease, such as rut, crack, and the comprehensive index PCI technology seriously weakened the preventive maintenance of pertinence and accuracy.

\subsection{Initial construction of evaluation index system}

In view of the complexity of asphalt pavement, the selection of preventive maintenance evaluation index should not only integrate the selected index with the existing highway performance evaluation system, but also fully consider the particularity of asphalt pavement. If only pavement damage status index (PCI) is used to evaluate the timing of preventive maintenance, the results will be too rough, and the correlation between road performance index and disease condition and maintenance measures is not strong, which is not conducive to the refined decision of preventive maintenance.

Preventive maintenance on pavement maintenance measures before the damage was not damaged, should be considered: (1) the road asphalt pavement preventive maintenance should meet the basic precondition for preventive maintenance, pavement condition index PQI and the sub-index achieve good, (2) the particularity of asphalt pavement early mild disease, loose, rut, crack, etc.

According to the specification and relevant scholars' research, the "possible complete set of indicators" for the evaluation of preventive maintenance of asphalt pavement is obtained, as shown in Table 5.

Table 5. Possible complete set of evaluation indicators.

\begin{tabular}{|l|l|}
\hline \multirow{4}{*}{ Macro technical } & $\begin{array}{l}\text { Pavement technical condition index } \\
\text { PQI }\end{array}$ \\
\cline { 2 - 2 } & $\begin{array}{l}\text { Pavement structure strength index } \\
\text { PSSI }\end{array}$ \\
\hline \multirow{4}{*}{ Micro technical } & cracks \\
\cline { 2 - 2 } & loose \\
\cline { 2 - 2 } & rut \\
\cline { 2 - 2 } & flushing \\
\hline \multirow{4}{*}{ Economic } & Unit maintenance cost \\
\cline { 2 - 2 } & The service life \\
\cline { 2 - 2 } & Times of major and medium repairs \\
\hline \multirow{2}{*}{ Environnemental } & Maintenance and energy consumption \\
\cline { 2 - 2 } & Carbon emissions \\
\hline
\end{tabular}

\subsection{Analysis and select of evaluation indicators}

The analysis and selection of preventive maintenance evaluation index should include the following characteristics: the index is easy to detect, it can represent pavement performance, and the index value changes with the adoption of preventive maintenance measures.

Combined with domestic and foreign research results showed that the comprehensive evaluation index is the base of asphalt pavement performance, under its guarantee compliance, and the selection of the single index to carry on the reasonable and effective evaluation, which can realize real and effective grasp of actual road conditions, and accordingly makes the corresponding road surface quality evaluation, so as to determine whether or how to make scientific and effective maintenance measures. Therefore, based on the comprehensive evaluation index, this paper puts forward a scientific and reasonable preventive maintenance system combining the comprehensive evaluation index as the objective standard and the sub-index as the micro standard.

(1) Pavement damage index: refers to a can truly reflect the physical characteristics of pavement damage due to the influence of objective factors, such as: vehicle load, geographical environment, construction factors, such as pavement damage showed a variety of forms, mainly ruts and cracks both damage is the most common, 
so in this study, sets the pavement damage single evaluation index to crack, rut.

(2) Pavement functional index: It mainly reflects a comfortable, safe and economic experience provided by the vehicle in the driving process through service level and driving quality. The pavement function mainly reflects the real condition of the pavement by means of flatness, rutting and anti-skid property. The corresponding indexes can be selected according to the specifications. The integral index IRI is taken as a single evaluation index of the driving quality of the pavement. The transverse force coefficient (SFC) is used as a single evaluation index for the safety performance of asphalt pavement.

(3) Pavement structure bearing capacity and pavement materials evaluation indexes: at present our country specification on pavement deflection as validation indexes of the bearing capacity of pavement structure in this paper, we explore the pavement performance index can reflect the real road conditions, real and effective for judgment basis to formulate reasonable, scientific and effective preventive maintenance measures, the pavement structure bearing capacity and pavement material attribute evaluation index in evaluating this but much of the.

\section{Conclusion}

In the paper, two simple and easy indicators were added, , on the basis of studying the current domestic maintenance specifications and relevant studies, and a relatively scientific and complete evaluation index system was established. The index considered the actual situation of preventive maintenance of asphalt pavement, providing reference for the decision making of preventive maintenance of asphalt pavement.

\section{References}

1. JTG 5421-2018.Specifications for Maintenance Design of Highway Asphalt Pavement[S]

2. JTG 5142-2019. Technical Specification for maintenance of Highway Asphalt Pavement[S]

3. Shi Yan, Ling Tian-qing, Cui Li-long, Ge Hao Chen Qiao-qiao. Study on Evaluation Standard for Asphalt Pavement Preventive Maintenance and Decision-making Optimization [J].Journal of Highway and Transportation Research and Development .2020.(10):25-34

4. Fan Xu-ying, Gao Feng-chun, Wang Hai-long, Li Yuzhong. A Model for Asphalt Pavement Preventive Maintenance Evaluation Based on Improved EWAHP [J]. Journal of Highway and Transportation Research and Development.2017(9):8-13

5. Marshal Dong et al. Time Decision Optimization of asphalt pavement pre-maintenance based on life Cycle [J]. Highway.2020(4):325-330

6. Zhang Chu nan, Tian Zhi peng. Based on preventive maintenance decision research of asphalt pavement of high grade highway. Road Engineering.2019(12):77-
80

7. Gao Feng chun. Research on preventive maintenance Evaluation and Maintenance Timing of Asphalt pavement in Zhangjiakou region [D].Hebei Institute of Architecture and Engineering.2019

8. Chen Sidi. Research on pavement preventive maintenance Decision Model based on Multiobjective particle swarm optimization algorithm [D].Beijing jiaotong university. 2019 